\title{
Extended Study on the Performance Evaluation of ISP MBG based Route Optimization Scheme in Mobile IPv4
}

\author{
sherif kamel
}

\begin{abstract}
Mobile IP has seen slow deployment for two major reasons; the need for enhancing edge routers with Home Agent/Foreign Agent functionality and the fact that triangle routing in such systems is not efficient. Triangle Routing is defined as the route that must be taken through the Home Agent for any traffic sent by the Correspondent Node to the Mobile Node. This route is triangle in nature and longer than the normal path between the Corresponded Node and the Mobile Node. Many protocols and research efforts have been developed to solve this problem.

Internet service Provider (ISP MBG) is one of the proposed techniques used for solving the triangle routing problem in conventional Mobile IPv4. This paper will provide a further study on the performance evaluation of Route Optimization in Mobile IPv4 based on ISP MBG scheme.

The proposed technique has been implemented and tested on the Microsoft.net platform. Simulation results prove that the new framework has solved the Triangle Routing Problem in Mobile IP by providing a shorter route with a minimum transmission time for all the datagrams transferred between the Correspondent Nodes and the Mobile Nodes.
\end{abstract}

Keywords: Mobile IP, Triangle Routing Problem, Route Optimization, Internet Service Provider, Point of Presence, Mobile IP Border Gateway, PoPs Virtual Network.

\section{Introduction}

Today, the number of wireless and mobile devices connected to the Internet is strongly growing. Wireless links and networks, mobile users and mobility related services form an increasing part of the internet infrastructure. These wireless and mobile parts are normally connected to larger, wired networks. Mastering the key concepts in mobile, wireless and wired technology areas are therefore of increasing importance in the society of today. Mobile IP is an internet protocol, defined by the Internet Engineering Task Force (IETF) that allows users keep the same IP address, and stay connected to the internet while roaming between networks. The key feature of Mobile IP design is that all required functionalities for processing and managing mobility information are embedded in well defined entities, the Home Agent (HA), Foreign Agent (FA), and Mobile Nodes (MNs) [1, 2]. When a MN moves from its Home Network (HN) to a Foreign Network (FN), the corret delivery of packets to its current point of attachment depends on the MN's IP address, which changes at every new point of attachment. Therefore, to ensure packets delivery to the MN, Mobile IP (MIP) allows the MN to use two IP addresses: The Home address and Care-of-Address (CoA). The home address is static and assigned to the $\mathrm{MN}$ at the $\mathrm{HN}$; CoA on the other hand is dynamic and represents the current location of the MN [2].

Original MIP has many problems such as home agent faults tolerance [3], HA overloading, and triangle routing problem. Triangle routing problem is considered as one of the main problems facing the implementation of MIP. When a Corresponding Node $(\mathrm{CN})$ sends traffic to the MN, the traffic gets first to the HA, which encapsulates this traffic and tunnels it to the FA. The FA de-tunnels the traffic and delivers it to the MN. The route taken by this traffic is triangular in nature, and the most extreme case of routing can be observed when the $\mathrm{CN}$ and the $\mathrm{MN}$ are in the same subnet. Many protocols have been invented to solve the triangle routing problem, such as forward tunneling and binding cache [], bidirectional route optimization [], the smooth handoff technique [5], a virtual home agent [6], and a port address translation based route optimization scheme [7]. Also, Kumar et al. [8] presented a route optimization technique in which the tunneling is done at one level above the HA in a hierarchical network instead of tunneling at the HA.

This paper provides an extended study on the performance of (ISP MBG) based route optimization in Mobile IPv4 [9]. This study is based on changing the system parameters including number of nodes, number of zones and number of pops serving each zone and check the performance of this scheme compared with conventional Mobile IP scheme. The paper is divided into 6 sections. Section 2 presents some basic concepts about Mobile IP while Section 3 introduces the concept of the Triangle Routing Problem in Mobile IP. Section 4 presents the ISP MBG technique used to optimize the Triangle Routing Problem in the Conventional Mobile IP technique. Section 5 introduces the analysis and evaluation of the proposed ISP MBG technique compared with the conventional Mobile IP technique. Finally section 6 presents the concluding remarks and the future work. 


\section{Mobile IP}

Mobile IP is a modification to IP that allows nodes to continue to receive datagrams no matter where they happen to be attached to the Internet. It involves some additional control messages that allow the IP nodes involved to manage their IP routing tables reliably. Scalability has been a dominant design factor during the development of Mobile IP, because in the future a high percentage of the nodes attached to the Internet will be capable mobility $[10,12]$.

\section{1 Mobile IP Terminologies}

Concerning the Mobile IP a set of terminologies are considered and defined as follows:

\begin{tabular}{|c|c|}
\hline Mobile Node (MN) & $\begin{array}{l}\text { a host or router that changes its point of attachment from one network or } \\
\text { subnetwork to another }\end{array}$ \\
\hline Home address (Ha) & $\begin{array}{l}\text { an IP address that is assigned for an extended period of time to a Mobile } \\
\text { Node in the Home Network. }\end{array}$ \\
\hline Home Agent (HA) & $\begin{array}{l}\text { a router on a Mobile Node's Home Network which tunnels datagrams } \\
\text { for delivery to the Mobile Node when it is away from home, and } \\
\text { maintains current location information for the Mobile Node. }\end{array}$ \\
\hline Home Network (HN) & $\begin{array}{l}\text { a network, possibly virtual, having a network prefix matching that of a } \\
\text { Mobile Node's Home Address. }\end{array}$ \\
\hline Foreign Agent (FA) & $\begin{array}{l}\text { a router on a Mobile Node's Visited Network which provides routing } \\
\text { services to the Mobile Node while registered. The Foreign Agent de- } \\
\text { tunnels and delivers datagrams to the Mobile Node. }\end{array}$ \\
\hline $\begin{array}{l}\text { Foreign Network } \\
\text { (FN) }\end{array}$ & any network other than the Mobile Node's Home Network. \\
\hline Care-of-Address (CoA) & $\begin{array}{l}\text { the termination point of a tunnel toward a Mobile Node, for datagrams } \\
\text { forwarded to the Mobile Node while it is away from home. }\end{array}$ \\
\hline
\end{tabular}

\begin{tabular}{|l|l|}
\hline $\begin{array}{l}\text { Correspondent } \\
\text { Node }(\mathbf{C N})\end{array}$ & $\begin{array}{l}\text { a peer with which a Mobile Node is communicating, it may be } \\
\text { either mobile or stationary. }\end{array}$ \\
\hline Link & $\begin{array}{l}\text { a facility or medium over which nodes can communicate at the } \\
\text { link layer. A link underlies the network layer. }\end{array}$ \\
\hline Node & a host or a router \\
\hline Tunnel & the path followed by a datagram while it is encapsulated \\
\hline Virtual Network & $\begin{array}{l}\text { a network with no physical instantiation beyond its router } \\
\text { (with a physical network interface on another network). }\end{array}$ \\
\hline Visited Network & $\begin{array}{l}\text { a network other than a Mobile Node's Home Network to } \\
\text { which the Mobile Node is currently connected. }\end{array}$ \\
\hline Visitor List & the list of Mobile Nodes visiting a Foreign Agent. \\
\hline Mobile Binding & $\begin{array}{l}\text { the association of Home Network with a Care-of-Address, } \\
\text { along with the remaining lifetime of that association }\end{array}$ \\
\hline
\end{tabular}

\section{2 Operation of Mobile IP}

Mobile IP is doing the following three relatively separate functions: Agent Discovery, Registration and Tunneling $[10,11]$.

\section{2. 1 Agent discovery}

The discovery process in Mobile IP is very similar to the router advertisement process defined in Internet Control Message Protocol (ICMP). For the purpose of discovery, a router or another network node that can act as an agent periodically issues a router advertisement ICMP message with an advertisement extension [10, 11].

\subsection{Registration}

Once a Mobile Node has recognized that it has transferred on a Foreign Network and has acquired a Care-of-Address, it needs to alert a Home Agent on its Home Network and requests that the Home Agent forwards its IP traffics. The registration process involves four steps: Registration Request to Foreign Agent, Foreign Agent Relays the Request to Home Agent, Registration Reply from the Home Agent to the Foreign Agent and finally the Foreign Agent Relays the Reply to the Mobile Node [10, 11].

\subsubsection{Tunneling}


Once a Mobile Node is registered with a Home Agent, the Home Agent must be able, to intercept IP datagrams sent to the Mobile Node's Home Network so that these datagrams can be forwarded via tunneling.

In the most general tunneling case as shown in Figure 1; the source, the encapsulator, the decapsulator and the destination are separate nodes. The encapsulator node is considered the entry point of the tunnel, while the decapsulator node is considered the exit point of tunnel. Multiple source-destination pairs can use the same tunnel between the encapsulator and decapsulator $[10,11]$.

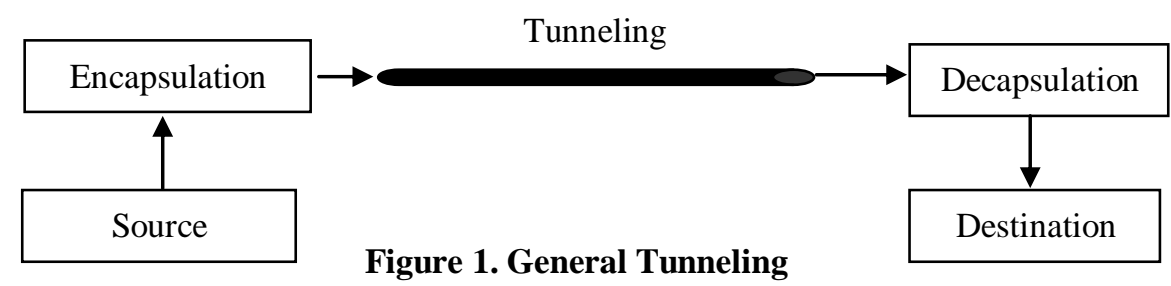

Three options for encapsulation (tunneling) are available for use by the Home Agent on behalf of the Mobile Node mainly: IP-ln-IP Encapsulation, Minimal Encapsulation, and General Routing Encapsulation (GRE).

\section{3 Mobile IP Operation Sequence}

With the three relatively separated functions; Agent Discovery, Registration and Tunneling; a rough outlines of the operation of Mobile IP Protocol is described as shown in Figure 2 [12].

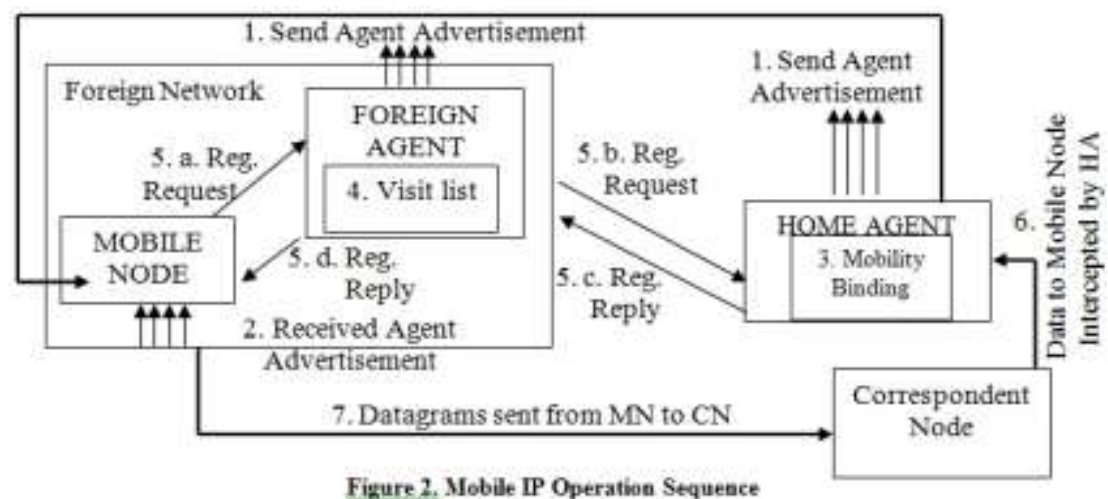

Mobile agents (Foreign Agents and Home Agents) advertise their presence via agent-advertisement messages. A Mobile Node receives an agent advertisement and determines whether it is on its Home Network or a Foreign Network. When the Mobile Node detects that it is located on its Home Network, it operates without mobility services. When a Mobile Node detects that it has moved to a Foreign Network, it obtains a CoA on the Foreign Network. The CoA can be either a Foreign Agent CoA or a Co-located CoA, then the Mobile Node registers its new CoA with its Home Agent through the exchange of a registration request and registration reply message, possibly by way of a Foreign Agent. Datagrams sent to the Mobile Node's Home Network are intercepted by its Home Agent, tunneled by the Home Agent to the Mobile Node's CoA, received at the tunnel endpoint (either at a Foreign Agent or at the Mobile Node itself), and finally delivered to the Mobile Node. In the reverse direction, datagrams sent by the Mobile Node may be delivered to their destination using standard IP routing mechanisms, without necessarily passing through the Home Agent.

\section{Triangle Routing Problem}

One of the basic problems facing the implementation of Mobile IP is the Triangle Routing Problem, since all the traffics between $\mathrm{CN}$ and MN should have to pass through a longer path than the normal one. This section introduces the definition and the drawbacks of the Triangle Routing Problem as shown in the following subsections.

\section{1 Triangle Routing Definition}

Triangle Routing Problem is considered as one of the problems facing the implementation of Mobile IP.

When a $\mathrm{CN}$ sends traffics to a $\mathrm{MN}$, the following sequence must be done:

1. Packets first get the HA.

2. Home Agent encapsulates these packets and tunnels them to the FA.

3. The Foreign Agent de-tunnels the packets and delivers them to the Mobile Node. 
As shown in Figure 3, the route taken by these packets is triangle in nature, and the most extreme case of routing can be observed when the Correspondent Node and Mobile Node are in the same subnet [13].

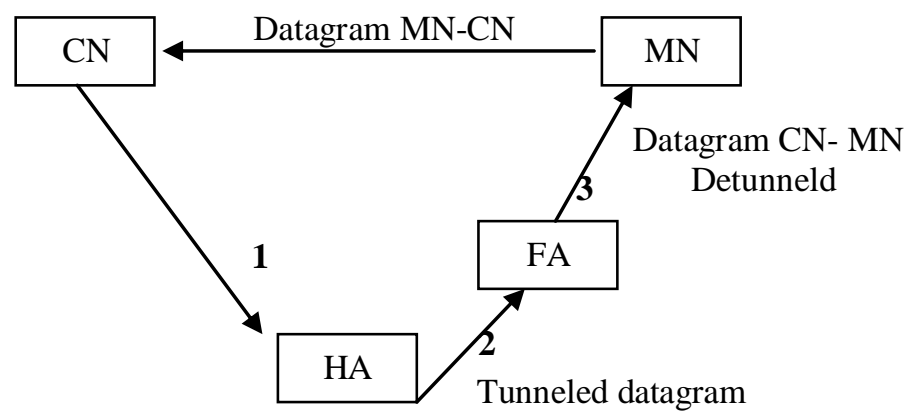

Figure 3. Illustration of the Triangle Routing Problem in Mobile IPv4

\section{2 Triangle Routing Drawbacks}

Conventional Mobile IP technique allows transparent interoperation between Mobile Nodes and their Correspondent Nodes, but forces all datagrams for a Mobile Node to be routed through its Home Agent. Thus, datagrams to the Mobile Node are often routed along paths that are significantly longer than optimal. This indirect routing can significantly delay the delivery of the datagrams to Mobile Nodes, and it places an unnecessary burden on the networks and routers along its path through the internet. The Triangle Routing drawbacks can be mentioned as follows:

1. Increases the delays per packet in datagrams transferred to the Mobile Node.

2. Wastes the network resources.

3. Home Agent bottle neck.

4. Delimits the scalability of Mobile IP protocol.

\section{ISP MBG Route Optimization Technique}

Before discussing the ISP MBG scheme it is important to mention the types of communications in Mobile Networks. In this issue, communication types involve the following [14]:

1. Communication between Mobile Node (MN) and Correspondent Node (CN) within the same Network; in this case the Home Agent receives a packet destined to the Mobile Node from a Correspondent Node and both of $\mathrm{MN}$ and $\mathrm{CN}$ are in the same network as shown in Figure 4.

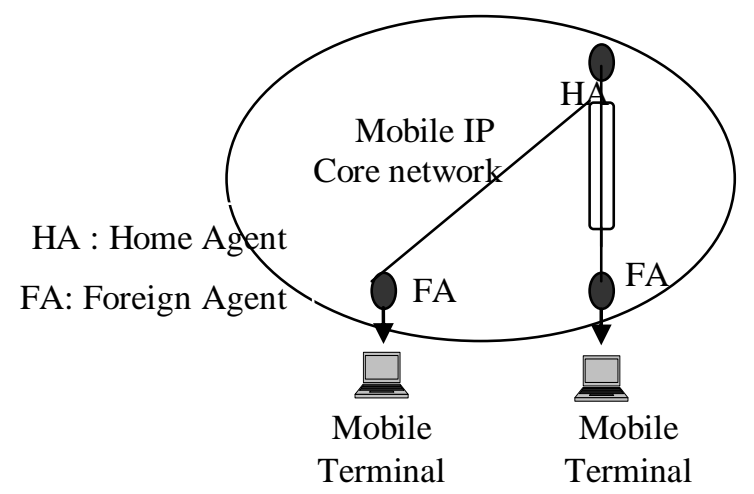

Figure 4. Connection between Two Mobile Terminals in the Same Network

2. Communication between Mobile Node (MN) and Corresponded Node $(\mathrm{CN})$ in two different Networks; when both of the Mobile Node and Correspondent Node are located in different networks as shown in Figure 5. It is supposed for the binding information to be transferred between the two networks and that will lead to a security related problem. To solve this problem, Mobile IP Border Gateways (MBGs); which are devices within the mobile networks; will maintain the binding information that must be added to the Correspondent Node without adding functions to terminals in the external networks [14, 15]. 


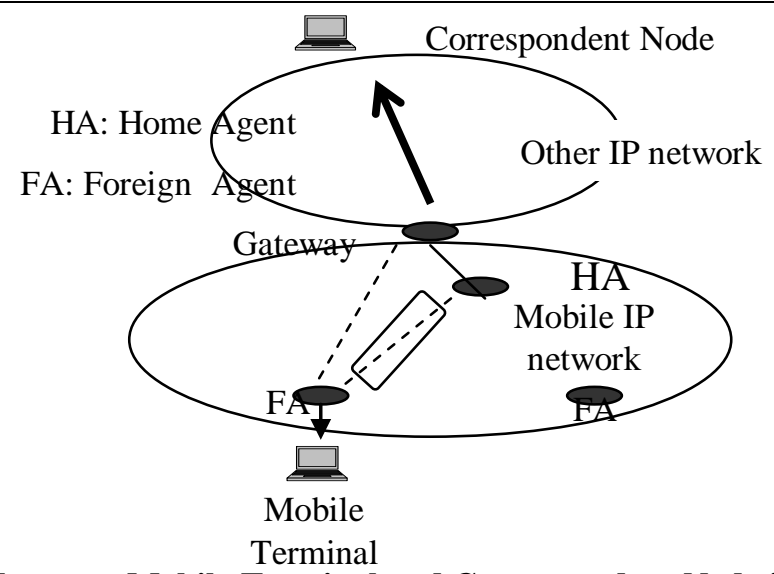

Figure 5. Connection between Mobile Terminal and Correspondent Node in two Different IP Networks

\subsection{Objectives of ISP MBG Technique}

The main objective of the proposed technique is to solve the Triangle Routing Problem in the Conventional Mobile IP technique. The proposed technique aims are:

1. Minimizing the average message delay.

2. Maximizing the network throughput (minimize the network blocking rate).

3. Using the network resources efficiently and eliminating the Home Agent (HA) processing bottleneck due to the fact that all communication from Correspondent Node $(\mathrm{CN})$ to Mobile Node $(\mathrm{MN})$ are necessarily routed through the Home Agent (HA).

4. Increasing the level of security between different networks by using the Mobile IP Border Gateway (MBG). This is important for maintaining the information that being used by the Correspondent Node $(\mathrm{CN})$ such as incoming packets from the external network are tunneled or routed and delivered directly to the Mobile Node (MN) instead of routing through the Home Agent (HA).

\subsubsection{Architectural design of ISP MBG Technique [9]} Problem.

Figure 6 presents the overall design of the proposed ISP MBG technique for the Route Optimization

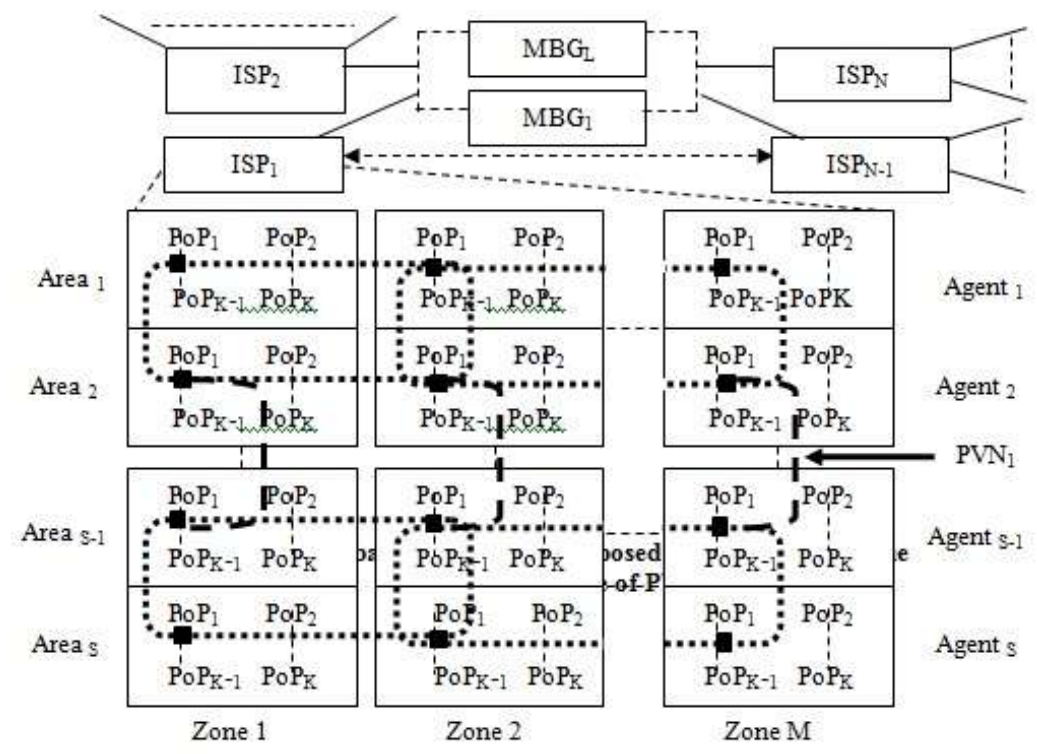

Figure 6. Global Views for the Proposed ISP MBG Technique with an Example of PVN

The design introduces the following:

1. Having a number of $\mathrm{N}$ Internet Service Providers $\mathrm{ISP}_{1}, \mathrm{ISP}_{2} \ldots \mathrm{ISP}_{\mathrm{N}}$ each covers a definite and different geographical place. They are separated by an L Mobile IP Border Gateways (MBGs) [14]. MBGs will maintain either the binding (Home address, Care-of-Address) or only the home information (Home address) for all the transferred nodes (Mobile Nodes) from one Internet Service Provider to another. That depends on whether we are using tunneling or routing technique to forward the traffics generated in one Internet 
Service Provider and destined to Mobile Node located in another Internet Service Provider. Also, using multiple MBGs will distribute uniformly the processing load among them.

2. Each Internet Service Provider is divided into a number of approximately $\mathrm{S}$ equal areas. Each area is served by an Agent that is considered as Home Agent for the nodes within that area and as Foreign Agent for all the nodes transferred from the other areas.

3. Each area is divided into multiple equal $\mathrm{M}$ zones where each zone is served by a fixed $\mathrm{K}$ equal number of Points-of-Presence (PoPs).

Figure 7 shows an example of the areas and PoPs classifications of each area for an Internet Service Provider.

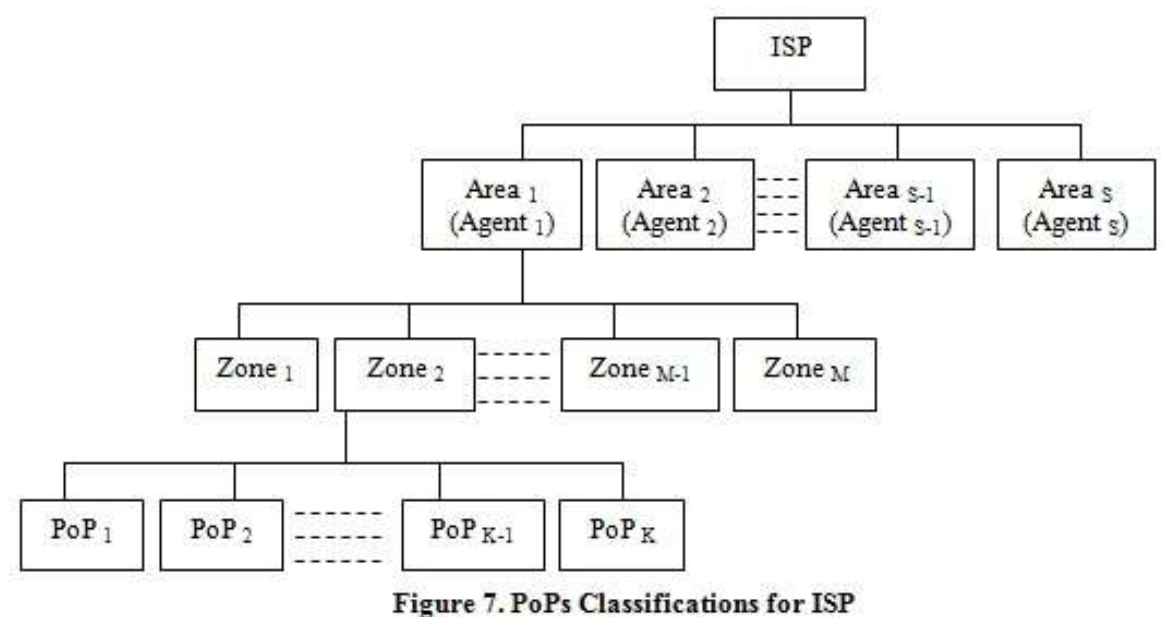

4. For each Internet Service Provider assuming that we have K PoPs virtual networks (PVNs) that can be placed in a fashion that is similar to (Ping-Pong) overlay network creation. This virtual network handles state information about all Mobile Nodes and Correspondent Nodes. For example, when a Mobile Node registers with one Points-of-Presence (PoPs), in one of the defined zones, the registration information will be available to all other zones through the PVN connecting that PoP with the other equivalent PoPs in the other zones. Figure 8 shows an example of PoPs Virtual Networks (PVNs) for ISP.

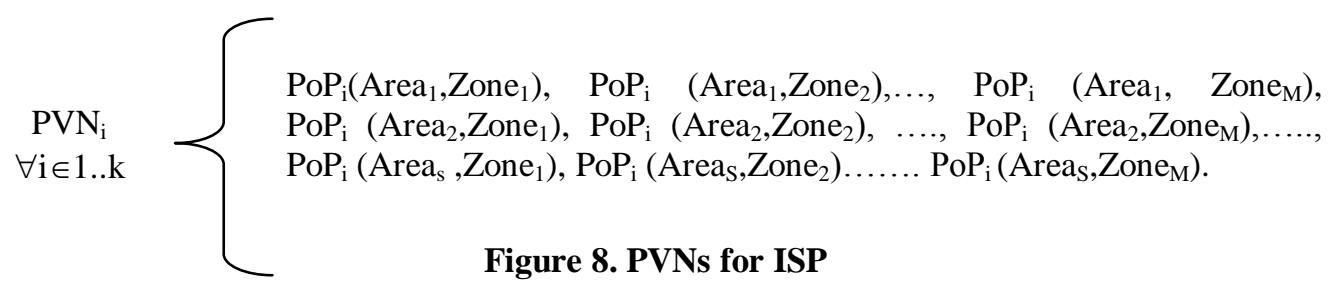

5. Each PoP serving a definite $\mathrm{X}$ number of nodes with a range $\mathrm{W}$ of addresses. The nodes that are within the same agent serving the PoP are called Local Nodes and those are in different agents, and transferred to the agent serving that PoP; are called External Nodes. The range of $\mathrm{W}$ addresses for each PoP is divided as follows:

1. A addresses for local nodes that are in service (Home address)

2. B addresses for local nodes that are in waiting (Home address)

3. C addresses for external nodes that are in service (Care-of- Address)

4. D addresses for external nodes that are in waiting (Care-of-Address)

\subsubsection{Sequences of ISP MBG Technique}

When a node is generated, it will be supported by the PoP serving its position, all of the home information concerning that node will be saved at that PoP. When a Mobile Node (MN) moves to another area or agent, the new agent will provide the node with the Care-of-Address. The home information for the node in the new position could be accessed through the PoP Virtual Networks (PVNs) that connects the node's home PoP to its new position serving the PoP. Figure 9 shows the operation sequence of the proposed technique.

The operation sequence for the proposed algorithm depends on whether both of the Correspondent Node and the Mobile Node are located in the same Internet Service Provider or both belong to different Internet Service Providers. So, when Correspondent Node needs to establish connection with the Mobile Node we have the following cases:

1. Both $\mathrm{CN}$ and $\mathrm{MN}$ belong to the same Internet Service Provider. 
a. $\mathrm{CN}$ connects to its home $\mathrm{PoP}\left(\mathrm{P}_{\mathrm{k}-1}\right)$ asking about the information for the Mobile Node.

b. The correspondent PoP searches its neighboring PoPs $\left(\mathrm{P}_{1}, \mathrm{P}_{2}, \ldots . \mathrm{P}_{\mathrm{k}-2}, \mathrm{P}_{\mathrm{k}}\right)$ in the same Zone, one of them is guaranteed to be connected to the virtual network of the Mobile Node.

c. The PoP which is connected to the Mobile Node's Virtual Network (i.e. $\mathrm{P}_{1}$ ) connects directly to the Mobile Node and the connection is established.

2. Both $\mathrm{CN}$ and MN belong to different Service Providers

a. The Mobile IP Border Gateway will keep the home information (Home address) for all the Mobile Nodes that are transferred form one ISP to another.

b. The Correspondent Node connects to its home PoP $\left(\mathrm{P}_{\mathrm{k}-1}\right)$ asking about the information for the Mobile Node.

c. The Correspondent PoP will ask its neighboring PoPs $\left(\mathrm{P}_{1}, \mathrm{P}_{2}, \ldots . \mathrm{P}_{\mathrm{k}-2}, \mathrm{P}_{\mathrm{k}}\right)$ about the Mobile Node's home information. One of the PoPs $\left(\mathrm{P}_{1}\right)$ is guaranteed to be connected to the virtual network of Mobile Node.

d. The PoP which is connected to the MN's Virtual Network (i.e. $P_{1}$ ) connects to MBG which has the original home information for the destined MN. MBG connects to the nearest PoP in the destination ISP which is connected to the new virtual network of Mobile Node

e. The connection is established between Correspondent Node and Mobile Node.

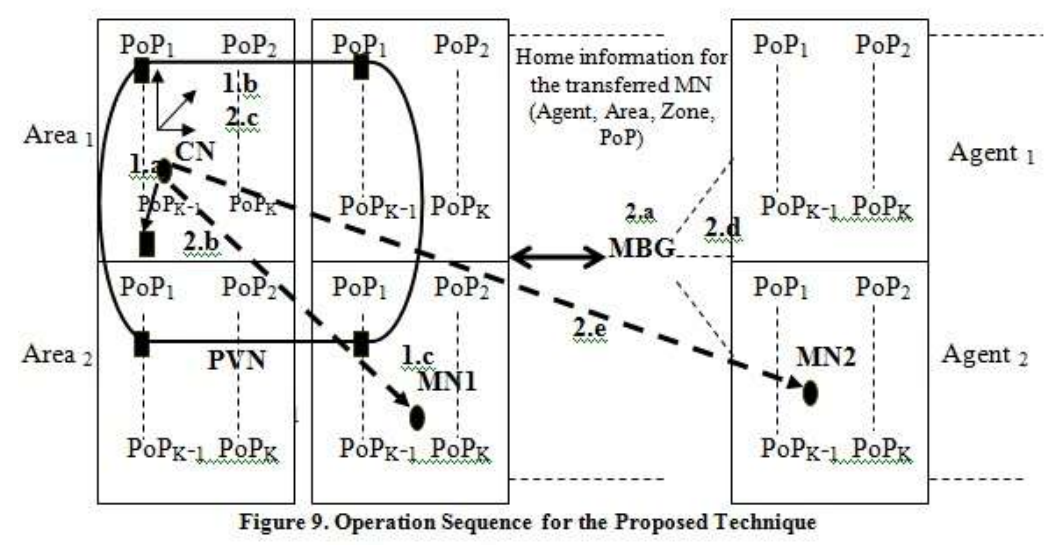

\section{Evaluation of ISP MBG Technique}

Simulation modeling is based on system programming such as data structures, flowcharts, programming languages and other tools that can be used to build up and characterizing system performance. For simplicity, it is preferable to depend on the simulation model.

\subsection{System Parameters}

The key point for establishing any system is to define its main parameters. This section introduces the simulation parameters, data structure and connection parameters for the designated system respectively in the following three subsections:

In [9] the simulation has been done for 2 similar ISPs each have 2 similar areas with 2 zones per each area and 4 pops serving each zone and for a total of 640 nodes. As an extended study for this technique, the new simulation is based on increasing the number of nodes for the total [600-2000] nodes and also number of zones and number of pops will be changed to 4 zones per area and 6 pops serving each zone. This study is to show how much the scheme is efficient even when the simulation parameters are changed to check the sustainability of ISP MBG technique as a route optimization technique for Mobile IP.

\subsubsection{Simulation Parameters}

A network with two internet service providers is considered, each with two similar areas. In our simulation there will be 2 structures one based an using 4 zones for each area and 4 pops serving each zone another structure based on using 2 zones for each area and 6 pops serving each zone.

Each PoP actually is serving 100 nodes with 150 addresses. The addresses are classified as 100 addresses for the nodes in service and 50 addresses for the nodes in waiting. The addresses for the nodes in service are classified as 75 addresses for the local generating nodes and 25 addresses for the externally generating nodes. By the same way for the addresses concerning the nodes in waiting. They are classified as 25 addresses for the local generating nodes and 25 addresses for the externally generating nodes. The addresses for the nodes are considered as integer numbers given to each node consecutively and depend on the location of the node, area, zone, PoP, and ISP it belongs to. Figure 10 shows an example of the nodes and address classification for $\mathrm{PoP}_{\mathrm{i}}$.

In the first structure the total number of zones all over the architecture design is 16 zones, each zone with 4 pops and the total number of pops is 64 . In the second structure, the total number of zones all over the 
Extended Study on the Performance Evaluation of ISP MBG based Route Optimization Scheme in architecture design is 8 zones. Each zone with 6 pops and the total number of pops is 48 . The total number of pops can be calculated by the following equation:

$$
\begin{aligned}
& \mathrm{P}_{\mathrm{N}}=\mathrm{P}_{\mathrm{K}} \times \mathrm{N}_{\mathrm{Z}} \quad \text { where; } \mathrm{P}_{\mathrm{N}} \quad \text { is the total number of PoPs } \\
& \mathrm{P}_{\mathrm{K}} \quad \text { is the number of PoPs within the Zone } \\
& \mathrm{N}_{\mathrm{Z}} \quad \text { is the total number of Zones }
\end{aligned}
$$
nodes.

Each PoP is serving 100 nodes, the simulation is done for a total number of nodes equals to $600-2000$

$$
\begin{aligned}
& \mathrm{N}_{\mathrm{T}}=\mathrm{P}_{\mathrm{N}} \times \mathrm{N}_{\mathrm{C}} \quad \text { where; } \quad \mathrm{N}_{\mathrm{T}} \text { is the total number of generating nodes } \\
& \mathrm{N}_{\mathrm{C}} \text { is the number of generating nodes in each connection. }
\end{aligned}
$$

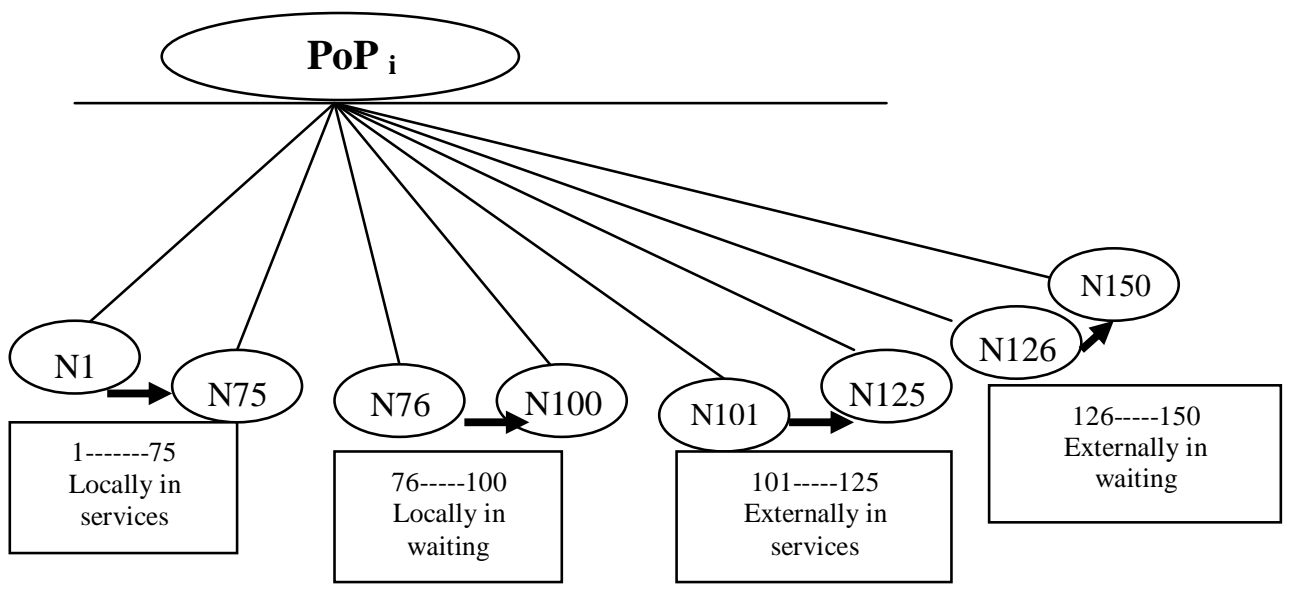

Figure 10: Nodes and Address Classifications for $\mathrm{PoP}_{\mathrm{i}}$

\subsubsection{Data structures}

The main data structures implemented in the simulation are: nodes, PoPs, ISPs, Areas, Agents, and Mobile IP Border Gateway (MBG). The class definitions and the structure definitions for all the data structures are implemented using C Sharp on the Microsoft.net platform.

\subsubsection{Connection parameters}

For the wireless communication design and implementation of the ISP MBG technique, the connection parameters for the implemented algorithm are the key point for running the program. The connection parameters can be classified as follows:

1. The distance in kilometers equivalent to the distance of 1 pixel.

2. Link speed for PoP connection

3. Link speed for Agents connection

4. PoP nodes count to serve.

5. PoP nodes count to wait

6. Agent nodes count to serve

7. Agent nodes count to wait

\subsection{System Construction}

Figure 11 shows the design architecture of the proposed ISP MBG techniques for 2 similar ISPs. Each PoP can be identified using the following three parameters respectively; ISP number, Agent number and Zone number:

$$
\begin{aligned}
P_{i}=P_{j, k, 1} \quad i=j, k, 1 \quad \text { where; } \quad P_{i}: \text { the } i^{\text {th }} & P o P \\
& j: \text { Internet Service Provider number } \\
& k: \text { Agent number } \\
& 1: \text { Zone number }
\end{aligned}
$$

Also, we have similar PoPs Virtual Networks (PVNs) for the two Internet Service Providers. Each PVN connects all similar POPs in all Zones, Areas, Agents, ISPs. Also in figure 11 we can change the number of agents, areas, zones and also the number of PoPs serving each zone based on the require parameters for comparison between the conventional Mobile IP and MBG ISP techniques. 


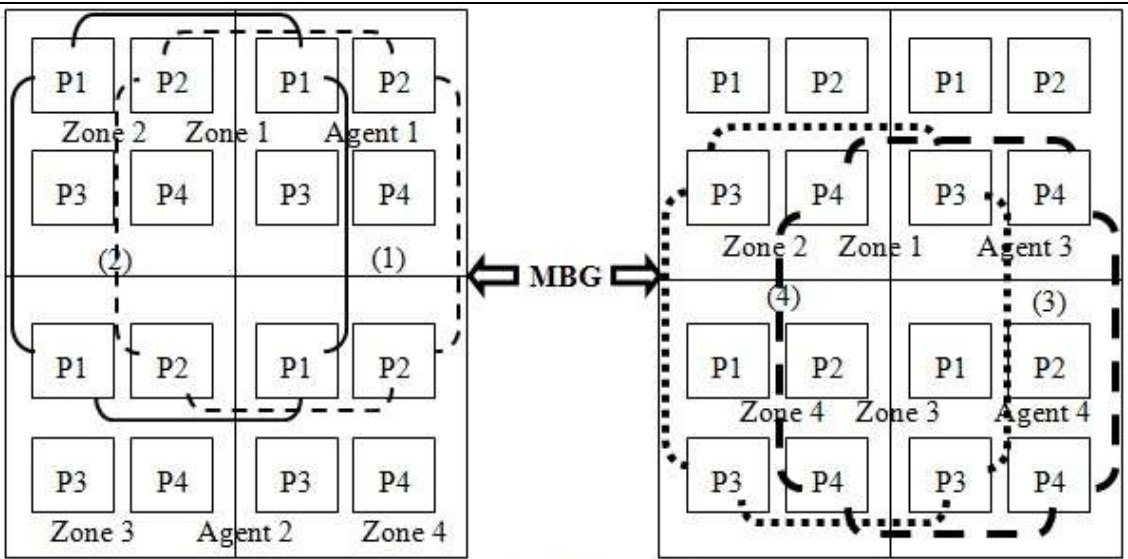

Figure 11. Design Architecture

\subsection{Performance Parameters}

To evaluate the performance of the ISP MBG technique, the following five measuring criteria are measured: Link Distance, Transmission Time, Blocking, Buffering and Security.

\subsubsection{Link distance}

The link distance is calculated in kilometers equivalent to the pixels distance between the two connecting nodes through the PoP links or the Agent links ( 1 pixel $=0.2 \mathrm{~km}$ ). The link distance can be calculated and formulated using the following equation:

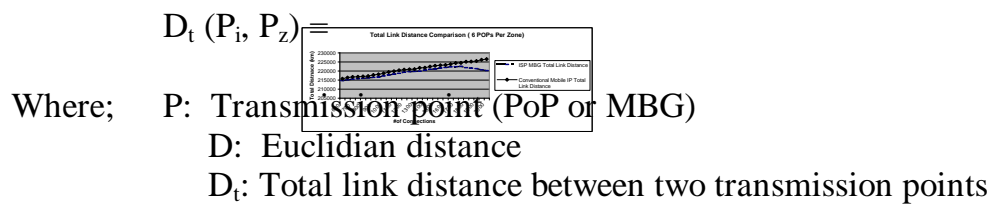

\section{3. 2 Transmission time}

Measuring the transmission time depends on the location of both Mobile Node (MN) and Correspondent Node $(\mathrm{CN})$ and whether both are located in the same area, same Internet Service Provider or either in different areas or different Internet Service Providers. The transmission time is calculated and formulated using the following general form of equation:

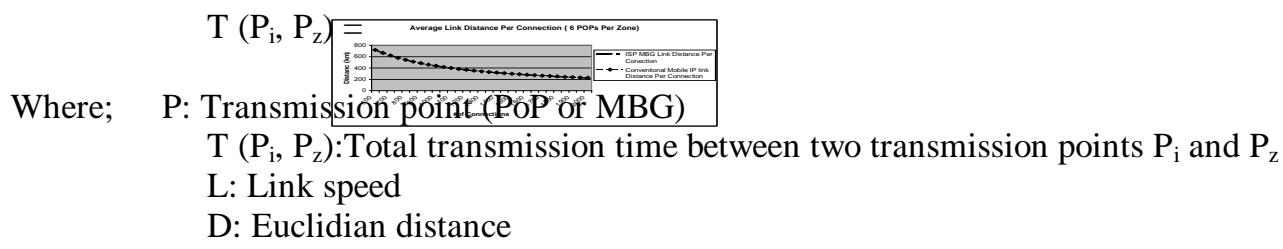

\subsubsection{Blocking}

Blocking is an important parameter to measure the overall performance of the network and its throughput. The blocking is measured as the number of blocked connections. Each connection has a pair of connecting nodes, (i.e. $\mathrm{N}$ connections $=2 \mathrm{~N}$ Nodes).

\section{3. 4 Buffering}

Buffering is considered as one of the network resources that must be optimally used. In the conventional Mobile IP technique, we have storage buffers for the agents whether they are Home Agents or Foreign Agents. In the proposed ISP MBG technique, each PoP has its own storage buffers which hold a limited number of nodes. The nodes are classified as nodes in service and nodes in waiting, with a range of addresses for the nodes that are either locally generated within the Agent (Home addresses) or imported from the other Agents. Using Point of Presence Virtual Network (PVN) will provide an efficient tool for accessing the Node's information between PoPs.[The measuring evaluation of this parameter is out of our scope].

\subsection{Security}

It is one of the rigid requirements for the performance evaluation between the conventional Mobile IP technique and the proposed one. It is measured as how much the technique itself provides a self-securing 

technique to protect the data transferred among the nodes located in different networks.[The evaluation of this parameter is out of our scope].

\subsection{Simulation Results}

The purpose of the simulation is to extend the study of the performance evaluation for ISP MBG technique compared with the conventional Mobile IP technique. The simulation results for the 2 similar ISPs, with 2 areas, 2 Zones per area and 4 PoPs per zone for the total number of 600 nodes has been discussed first [9]. For further study of the ISP MBG technique with different ISPs structures, the running for the implemented algorithm is done and calculated based on generating randomly a total number of nodes $\mathrm{N}=2000$ nodes in 15 steps, each step includes 100 more nodes (i.e 50 connections). In each step, the whole algorithm is executed, and the connection is also randomly done between each pair of nodes. To obtain real results, the algorithm is executed many times and the comparison is done based on the average values of the results.

\subsubsection{Simulation Result for 2 Similar ISPs [2 Areas, 2 Zones/Area, 4 PoPs/Zone] [9]}

\subsubsection{Simulation Results for Link Distance (total 600 nods, 20 nods per connection)}

Figures 12.a and 12.b show the total link distance and the average link distance per connection for both the proposed ISP MBG and the conventional Mobile IP technique respectively. It is clear that the proposed ISP MBG technique outperforms the conventional Mobile IP technique. The route taken by the conventional Mobile IP technique has to pass through the Home Agent which tunnels the data to the Foreign Agent. The route in the proposed ISP MBG technique is taken through the home PoP of CN and the PoP virtual network of MN which leads directly to the MN in case of one ISP. In case of using two Internet service Providers the route has to pass through MBG to the MN's PoP virtual network in the second ISP which leads directly to the MN.

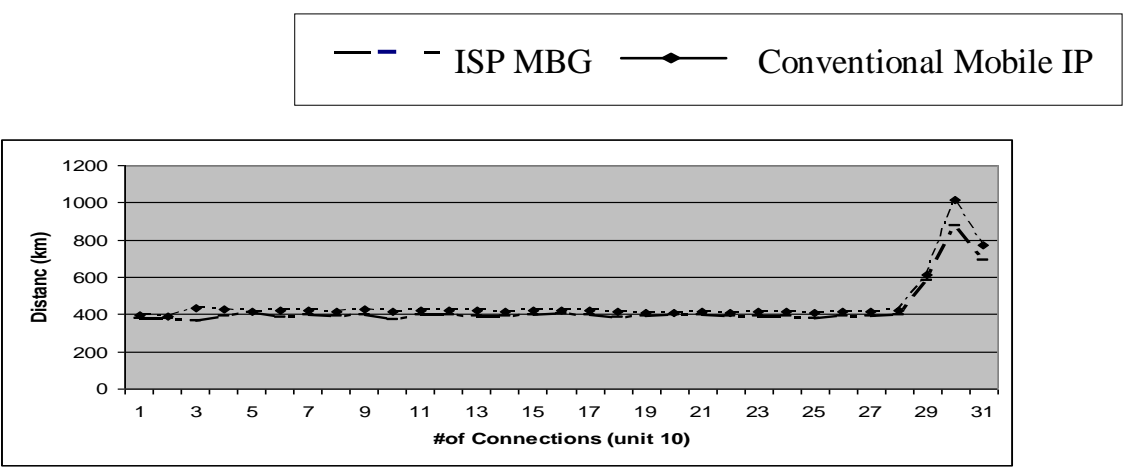

(a) Total Link Distance for the Conventional and ISP Techniqe
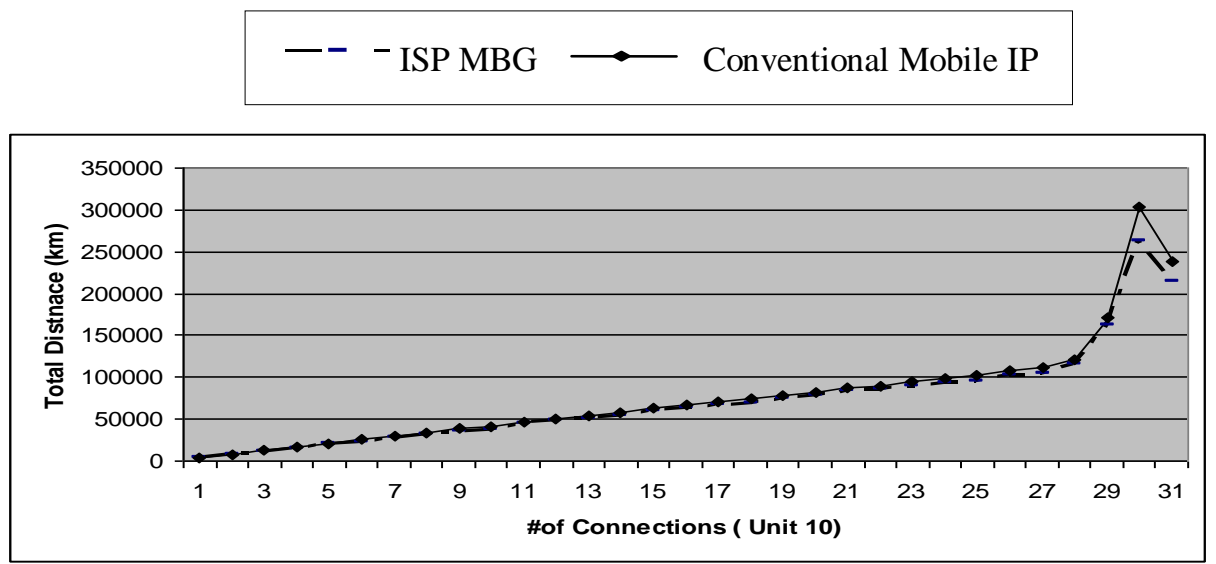

(b) Average Link Distances for the Conventional and ISP Techniqe

Figure 12. Link Distance Comparison

\subsubsection{Simulation results for transmission time}

Figure 13.a and 13.b show the total transmission time and the average transmission time (in seconds) per connection against the number of connections for both the conventional Mobile IP technique and the ISP MBG technique respectively. The figures show a great reduction in the transmission time using the proposed ISP MBG technique, compared with the conventional one. That result is expected because of using PoPs and PVNs: 
Extended Study on the Performance Evaluation of ISP MBG based Route Optimization Scheme in the home information for any node will be available anywhere among the networks and not only restricted on the Home Agents that could be far away from the connecting nodes.

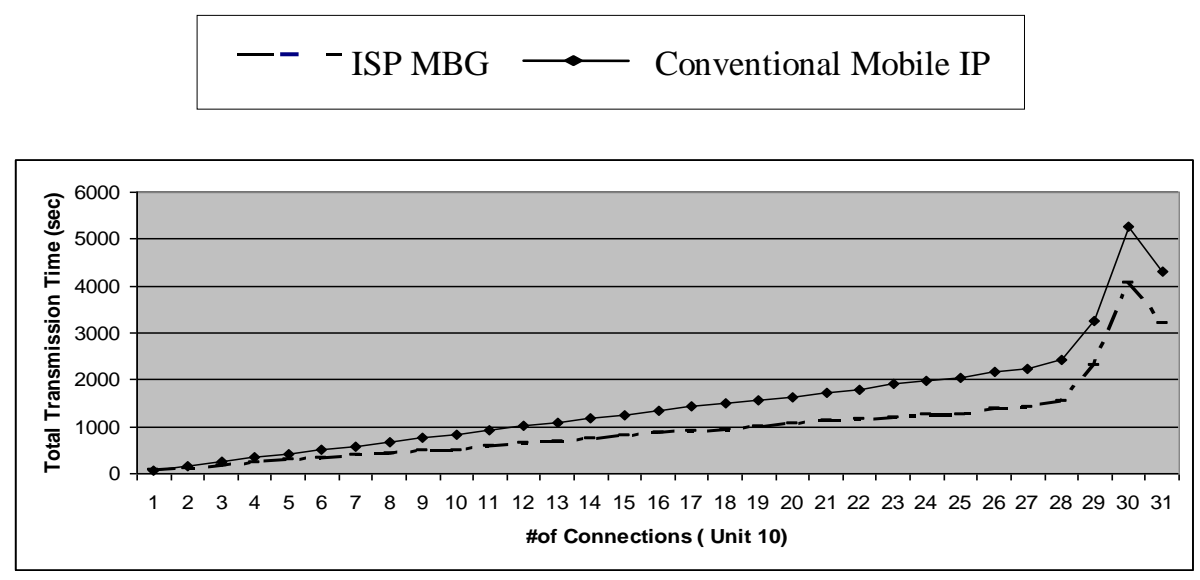

(a) Total Transmission Time for the Conventional and ISP Techniques

$$
\text { - - ISP MBG — Conventional Mobile IP }
$$

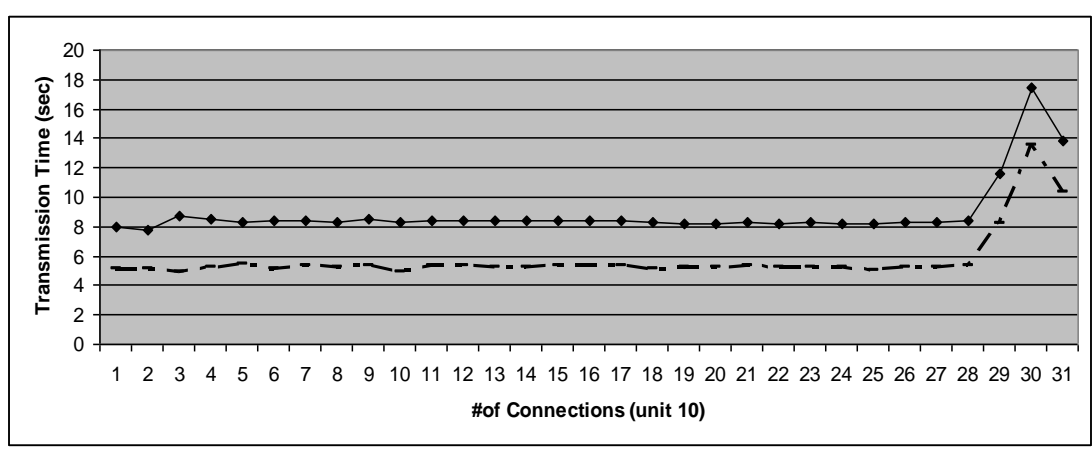

(b) Average Transmission Time for the Conventional and ISP Techniques Figure 13. Transmission Time Comparison

\subsubsection{Simulation Results for Blocking}

Figure 14 details the average number of blocking in both the conventional Mobile IP technique and the proposed one. Each blocked connection is considered as single pair of connecting nodes. The figure shows that as long as the number of connections $<28$ (i.e. number of nodes $<560, " 560=$ number of connections $\{28\} \times$ number of generating nodes in each connection $\{20\} ")$.

If the number of connection is increased (number of connections $\geq 28$ ) meaning that the number of nodes is also increased (number of nodes $\geq 560$ ) the blocking rate will be increased for both the conventional Mobile IP technique and the proposed one. The number of blocked connections using the newly proposed ISP MBG technique is less than that of the conventional Mobile IP technique. In the conventional Mobile IP technique, the HA is overwhelmed with an excessive amount of nodes' control messages compared with the proposed technique in which the control messages are divided among the PoPs. Each PoP covers a number of nodes and the virtual network between PoPs helps in getting the information easily. As the number of blocked connections decreased that leads to increase the number of paired successful connections which means the throughput will be increased. 


\section{$-\begin{aligned} & - \\ & -\end{aligned}$}

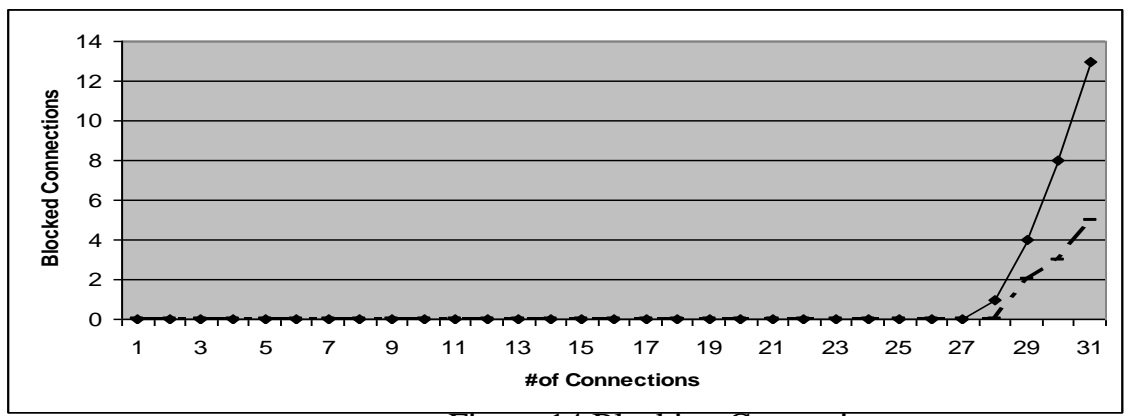

Figure 14 Blocking Comparison

5.4.2. Simulation Results for 2 Similar ISPs [ 2 Areas, 4 Zones/Area, 4 PoPs/Zone, Number of Nodes 6002000 Nodes.]

\subsubsection{Simulation Results for Link Distance}

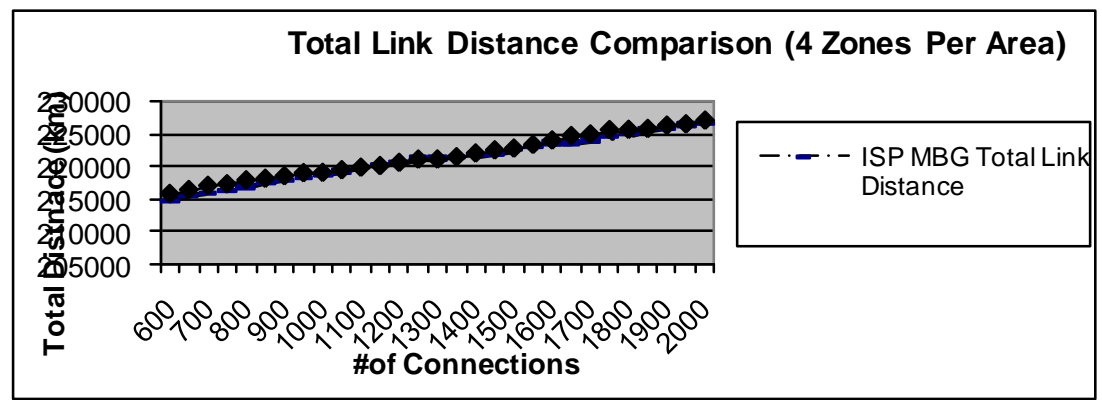

(a) Total Link Distance for the Conventional and ISP Technique

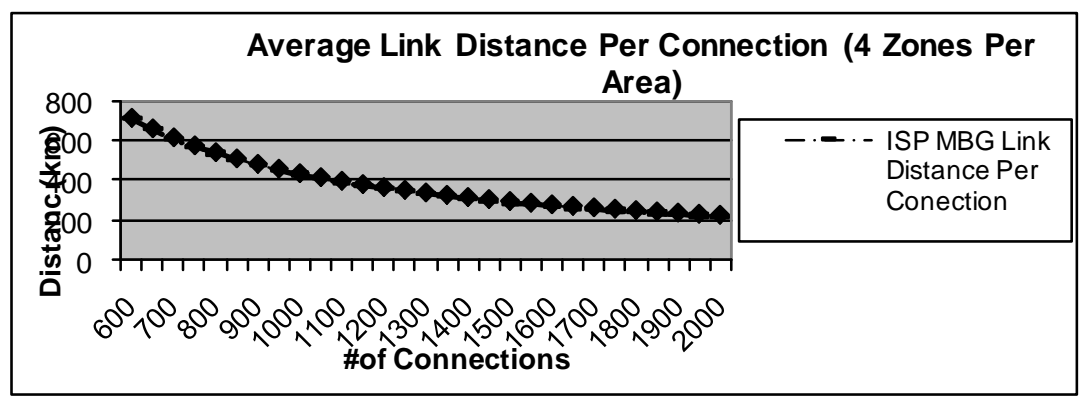

(b) Average Link Distance for the Conventional and ISP Techniqe Figure 15. Link Distance Comparison

Figure 15.a and 15.b show that when the number of zones per area increases, the link distance for the new technique increase due to redundancies in the virtual networks, but still it is better than the conventional one (although close on performance) 
Extended Study on the Performance Evaluation of ISP MBG based Route Optimization Scheme in 5.4.2.2. Simulation Result for Transmission Time

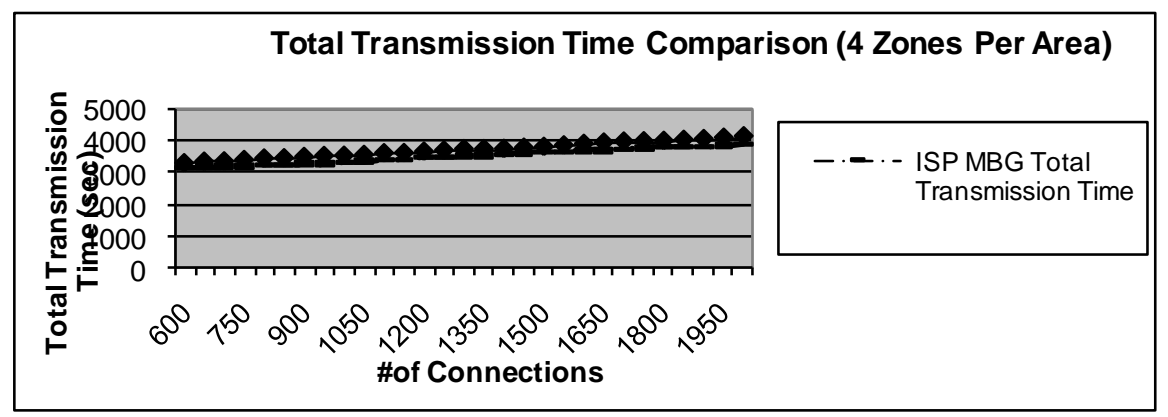

(a) Total Transmission Time for the Conventional and ISP Techniques

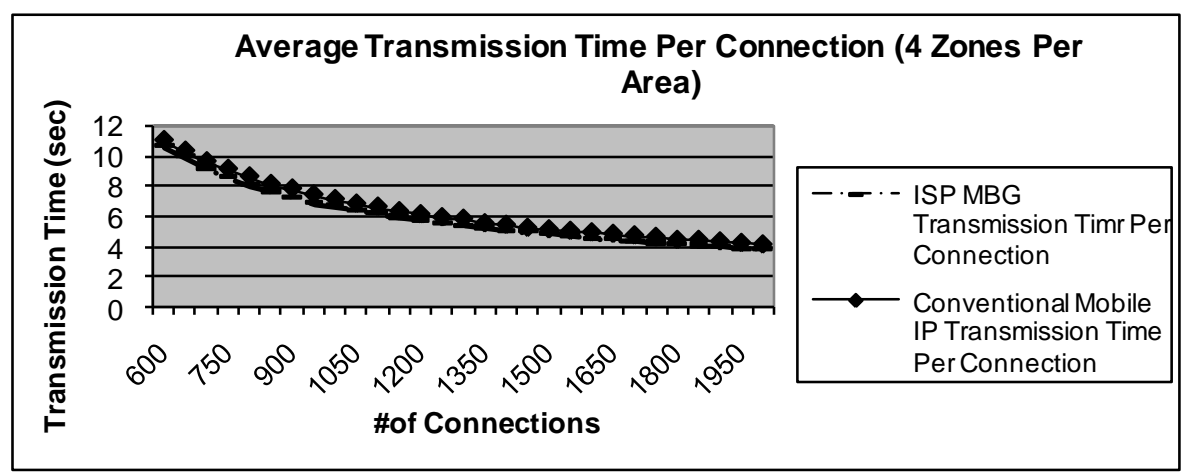

(b) Average Transmission Time for the Conventional and ISP Techniques Figure 16. Transmission Time Comparison

Figure 16.a and 16.b show that the transmission time required for both techniques are close to each other due to redundancies in the virtual networks.

\subsubsection{Simulation Result for Throughput}

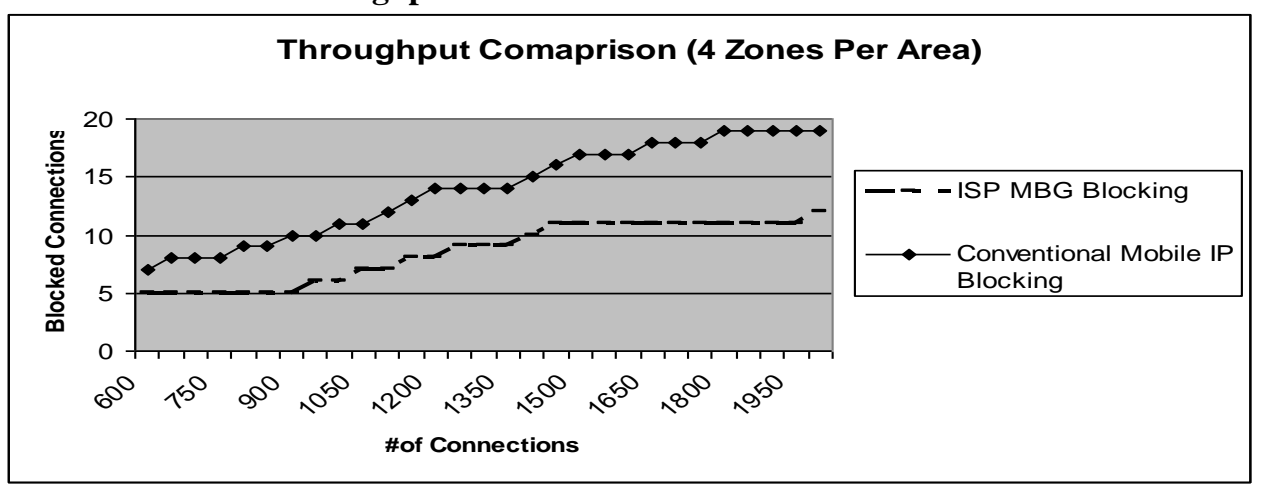

Figure 17. Throughput Comparison

Figure 17 show that he new technique still outperforms the conventional one in the storage and throughput, increasing number of zones increases the storage capabilities, so, less calls are blocked.

5.4.3 Simulation Results for 2 Similar ISPs [2 Areas, 2 Zones/Area, 6 PoPs/Zone, Number of Nodes 6002000 Nodes.] 
Extended Study on the Performance Evaluation of ISP MBG based Route Optimization Scheme in 5.4.3.1. Simulation Results for the link distance

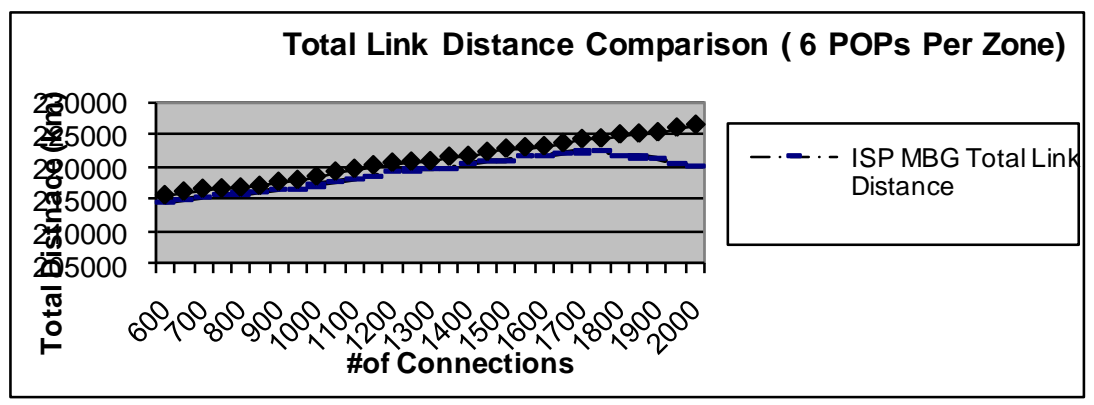

(a) Total Link Distance for the Conventional and ISP Technique

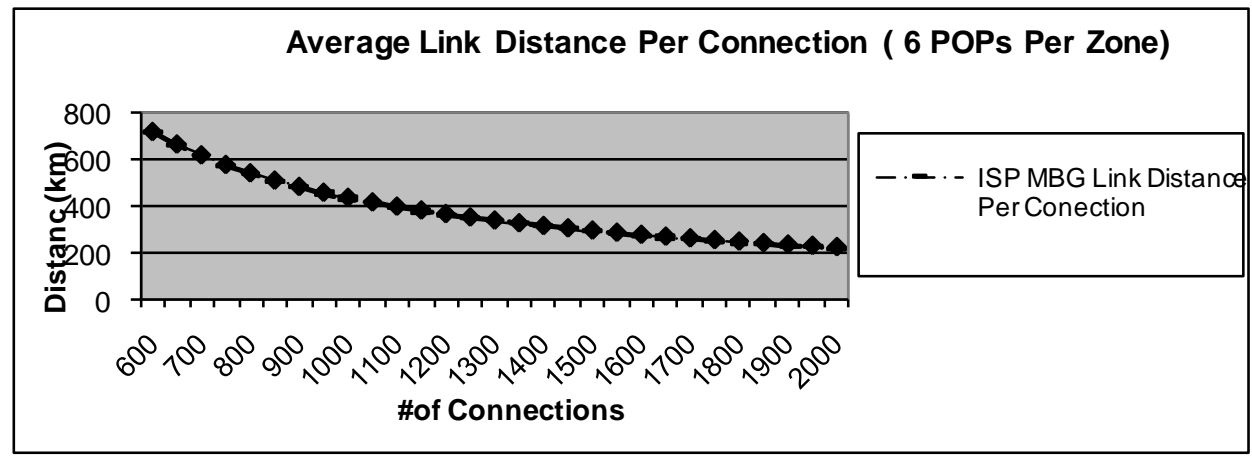

(b) Average Link Distance for the Conventional and ISP Techniqe

Figure 18. Link Distance Comparison

Figure 18.a and 18.b show that when increasing number of pops per zone the link distance in the new technique is less than that of the old technique, although they are close on the average.

\subsubsection{Simulation Results for Transmission Time}

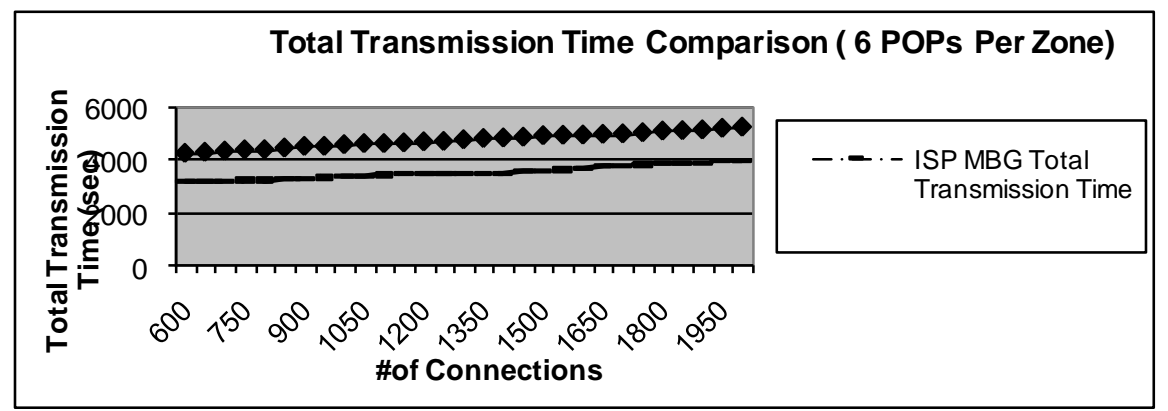

(a) Total Transmission Time for the Conventional and ISP Techniques

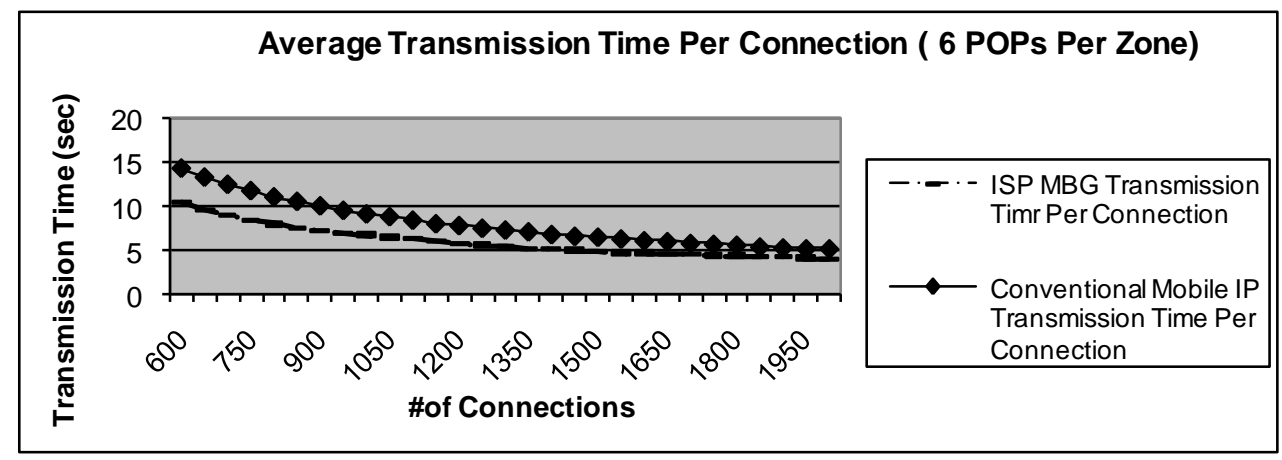

(b) Average Transmission Time for the Conventional and ISP Techniques

Figure 19. Transmission Time Comparison 
Figure 19.a and 19.b show that due to the increase of the POPs serving the zones, the calling node can reach the mobile node much faster than in the old techniques, and it outperforms the conventional techniques on the average transmission time.

\subsubsection{Simulation Result for Throughput}

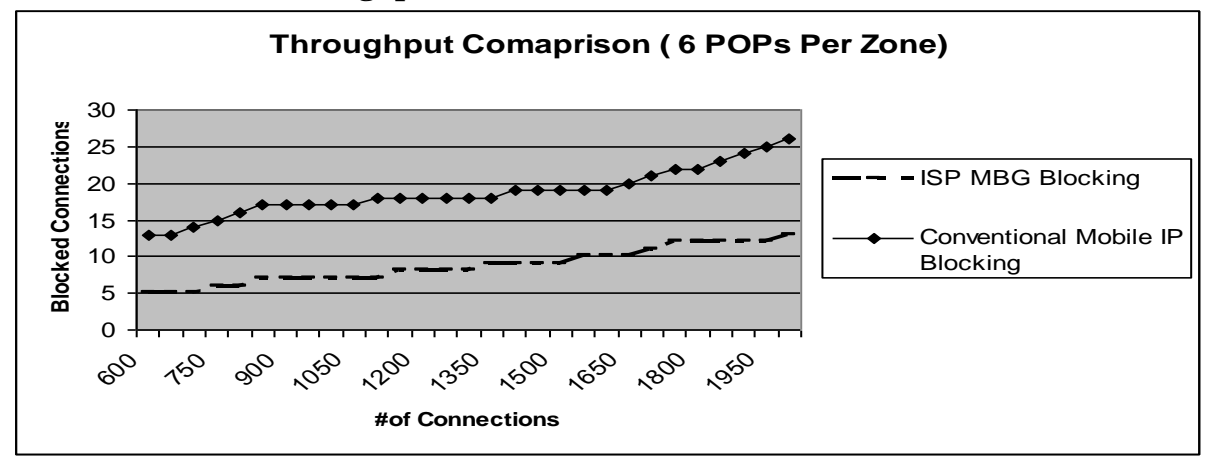

Figure 20. Throughput Comparison

Figure 20 shows that the throughput of new technique is still better than conventional techniques

\subsubsection{Buffering}

Concerning Buffering; it has been found that the proposed ISP MBG technique provides better buffering than that of the Conventional Mobile IP technique. This is due to the fact that using PVN with the proposed technique facilitates the process of handling and accessing information for the nodes in correspondences between PoPs without any redundancy. Comparatively, the conventional technique requires more buffers due to the redundancy of having a multiple copies of nodes' home information at each PoP of the Internet service Provider. This leads to less use of buffering storages than that of the conventional technique which needs storage buffer for each node to hold all of its information at each PoP. Also, as a cost wise, it has been found that the cost for the Agent's buffers is higher than that of the PoP's buffers

\subsubsection{Security}

The Security design has a great concern in Mobile IP. The proposed ISP MBG technique is considered as the self securing system. Using Mobile IP Border Gateway (MBG) will keep the information (Home address or binding information) for all Mobile Nodes crossing their network to another network. So, any CN in one network does not need to maintain any private external information concerning the new IP network where the MNs visit and all of the MN's information could be accessed directly through the Mobile Border Gateway. Comparatively, the conventional Mobile IP technique needs rigid requirements for the authentication to prevent the malicious users from interrupting the connection between $\mathrm{MN}$ and $\mathrm{CN}$ that maintains the binding information (Ha, CoA).

\section{Conclusion and Future Work}

In this paper a further study on ISP MBG route optimization technique has been introduced to check the performance evaluation of the technique when the simulation parameters [zones an pops] are changed.

The design of technique is based on using a number of Internet Service Providers (ISPs) separated by a Multiple Mobile IP Border Gateways (MBGs) which are used to keep the binding information or the home information for the transferred Mobile Nodes between ISPs. Each ISP is composed of an approximately a number of an equal areas, each is served by an agent and is composed of a multiple equal zones. Each zone is served by a definite number of Points of Presences (PoPs). Each PoP is serving a number of nodes with a range of addresses. Virtual Networks are used to connect the PoPs in such a way the redundancy in keeping the nodes information will be minimized or almost cancelled. The main function of the proposed technique is to get the shortest routing path for the packets transferred between the Correspondent Nodes and Mobile Nodes based on the PoPs information, PVN and the MBG.The simulated network design of our case study is based on using two Internet Service Providers separated by one Mobile IP Border Gateway. Each ISP is divided into two equal areas. The simulation is applied twice once based on using 4 zones per each area and 4 pops serving each zone. Another simulation is based on using 2 zones per area and 6 pops serving each zone.Each PoP is serving 100 nodes with a range of 150 addresses. The simulation results for the Link Distance, Transmission Time, Blocking, Buffering and Security show that the proposed (ISP MBG) technique outperforms the conventional Mobile IP technique by minimizing the Link Distance, Transmission Time, Blocking, Buffering. Also, it gives a higher level of security than that used with conventional Mobile IP technique. 
Extended Study on the Performance Evaluation of ISP MBG based Route Optimization Scheme in

This work can be considered applicable for the following: better performance related to the criteria of measuring parameters ,no addition of external hardware devices are required, more reliablility and flexibility of the simulation model, and scalability for using more PoPs and nodes .

For more further study on the performance evaluation of ISP MBG route optimization technique, further evaluation will be done based on using 2 different ISP structures.

\section{Reference}

[1] C. Perkins." IP Mobility Support for IPv4", IETF RFC 3344 Ed., Oct. 2002

[2] C. Smith and D. Collins, "3G Wireless Networks", "McGraw-Hill, United States, 2002.

[3] Y. Huang, and M. Chuang, "Fault tolerance for home agents in mobile IP", 2006.

[4] C. Wu, A. Cheng, S. Lee, J. Ho, and D. Lee, "Bi-directional Route Optimization in Mobile IP Over Wireless LAN", Institute of Information Science, Academia Sinica, Taiper, Taiwan, Vol. 2, pp 1168-1172. 2002.

[5] B. Ayani, "Smooth Handoff in Mobile IP", Master Thesis, University of California in Berkeley. May 2005.

[6] G. Qiang and A. Acampora, "A Virtual Home Agent based Route Optimization for Mobile IP", Wireless, 28 Sept. 2000.

[7] D. Badami, N. Thanthery, T. Best, R. Bhagvathula, R. Pendse, "Port Address Translation Based Route Optimization for Mobile IP", Vehicular Technology Conference, (5), PP. 3110-3114. 2004.

[8] C. Kumar, N. Tyagi, and R. Tripathi, "Performance of Mobile IP with New Route Optimization Technique". IEEE. International Conference, Institute of Engineering and Rural Technol, Allahadad, India, pp. 522-526. 2005.

[9] Sherif Kamel Hussein, Iman Saroit Ismail, S. H. Ahmed, "Solving the Triangle Routing Problem in Mobile IP", informatics journal, faculty of computers and information technology, Cairo University published issue, June 2006.

[10] C.Kumar, N.Tyagi , Tripathi R., "Performance of Mobile IP with new Route Optimization Technique", IEEE International Conference, Institute of Engineering and Rural Technol, Allahadad, India, PP. 522-526, January 23-25, 2005.

[11] D.Badami, N.Thanthry, T.Best, R.Bhagavathula and R.Pendse., "Port Address Translation based Route Optimization For Mobile IP", Vehicular Technology Conference, IEEE $60^{\text {th }}$, Department of Electrical and Computer Engineering, Wichita State University, KS, USA, Vol. 5, PP. 3110-3114, September 26-29, 2004.

[12] Moheb R. Girgis, Tarken, Mohamoud Youssef S. Takroni and Hassan S. Hassan, "Performance Evaluation of New Route Optimization Technique for Mobile ". IP International Journal of Netowrk Security \& Its Applications (IJNSA), Vol., No. 3, October 2009

[13] C. Perkins, "IP Mobility Support for IPV4", RFC 3344, Work on Progress, August 2002.

[14] Y.Takagi ,T.Ihara and H.Obnishi,"Mobile IP Route Optimization Method for Next Generation Mobile Networks", Electonicsand-Communications in Japan,Part 1,Vol.86,No.2 ,PP.31-41, February 2003.

[15] M.Caesar and J.Rexford, ,BGP Routing Policies in ISP Networks " IEEE Network, University of California and Princeton University ,PP.5-11,November/December 2005. 